\title{
Analysis of the flow-volume curve in children and adolescents with allergic rhinitis without asthma
}

\author{
Luciano Ianiero, M.D., ${ }^{a}$ Ricardo J. Saranz, M.D, ${ }^{a}$ Natalia A. Lozano, M.D., ${ }^{a}$ \\ Alejandro Lozano, M.D., ${ }^{a}$ Laura V. Sasia, M.D., ${ }^{a}$ Maximiliano Ramírez, M.D., ${ }^{b}$ and \\ Eduardo Cuestas, M.D., ${ }^{c}$
}

\begin{abstract}
Introduction. There is epidemiological, functional and pathologic evidence that relates upper and lower airways, clinically known as a single respiratory tract. Patients with allergic rhinitis without asthma may present subclinical abnormal spirometry parameters.

Objectives. To describe the results of the flowvolume curve in a group of patients with allergic rhinitis without asthma and analyze the possible associations between anthropometric, clinical and biochemical outcome measures with abnormal spirometry results.

Population and Methods. Observational, descriptive study including children and adolescents aged 6 to 18 years old with symptoms of allergic rhinitis without asthma. Age, gender, body mass index and duration of rhinitis were determined as per the subject's medical record. Allergen skin tests, flow-volume curve spirometry, determination of eosinophil count in blood and in nasal secretions, and total serum IgE were performed.

Results. A total of 84 patients were studied; 21 (25\%; 95\% CI: 15.1-34.8) presented at least one altered spirometry outcome measure. The FEV1/FVC ratio was the most affected outcome measure (10/84; $12 \%$; 95\% CI: 4.3-19.4). The multiple logistic regression analysis determined that spirometry alterations were associated with the number of blood eosinophils (OR: 1.00229; 95\% CI: 1.00022-1.00436; $\mathrm{p}=0.03$ ) and the body mass index (OR:1.31282;95\% CI: 1.08611-1.58685; $\mathrm{p}=0.0049$ ).

Conclusions. Our results showed spirometry alterations in a considerable percentage of children and adolescents with allergic rhinitis without asthma. The blood eosinophil count and the body mass index could be associated with a sub-clinical alteration of pulmonary function. Key words: allergic rhinitis, spirometry, pulmonary function tests.
\end{abstract}

http://dx.doi.org/10.5546/aap.2013.322

\section{INTRODUCTION}

Allergic rhinitis is one of the most common chronic diseases in pediatrics. Phase III of the International Study of Asthma and Allergies in Childhood (ISAAC) revealed that the prevalence of asthma in Argentina was 20.6\% in the 6-7 year old group, and $34.9 \%$ in the $13-14$ year old group. ${ }^{1}$

The relation between rhinitis and asthma has been demonstrated by epidemiological, clinical, functional and therapeutic evidence. ${ }^{2}$ These two conditions have a common pathophysiological link with atopy, and there is evidence indicating that eosinophil-mediated inflammation is involved in their pathogenesis. ${ }^{3}$ This has led to the holistic concept of a single disease with involvement of the respiratory tract as a whole. ${ }^{3,4}$

A high percentage of children with allergic rhinitis without asthma have bronchial hyperreactivity to methacholine, histamine and exercise, ${ }^{4-6}$ which could mean an increase in their risk of developing asthma. ${ }^{4}$ Recently, subclinical alterations have been found in the pulmonary function of adults ${ }^{7}$ and children with allergic rhinitis without asthma, with increased reactivity to bronchodilator response, ${ }^{10}$ which had not been observed in prior research including patients with rhinitis, chronic rhinosinusitis, and nasal polyposis. ${ }^{11-13}$

Such alterations would mainly compromise the forced expiratory flow between $25 \%$ and $75 \%$ of the forced vital capacity (FEF 25-75\%); for this reason, rhinitis guidelines 14 consider it an early marker of pathologic limitation of the peripheral airway, a criterion only partially shared by other authors, who assign it a relative functional value. ${ }^{15}$

Allergic airway inflammation can obstruct nasal airflow and cause subclinical bronchial involvement; 
therefore, our objectives were to describe the results of the flow-volume curve in a group of patients with allergic rhinitis without asthma and analyze possible associations between anthropometric, clinical and biochemical outcome measures with abnormal spirometry results.

\section{POPULATION AND METHODS}

Study with observational and descriptive design including 6 to 18 year old male and female children and adolescents who attended the Allergy and Immunology Service of the Clínica Universitaria Reina Fabiola in the City of Córdoba, Argentina, between December 30, 2011 and June 31, 2012 with a diagnosis of allergic rhinitis based on the presence of typical nasal symptoms (rhinorrhea, obstruction, pruritus and sneezing) and positive skin tests to at least one relevant allergen (whether perennial or seasonal). Patients with the following conditions were excluded:

a. A history of asthma or equivalent symptoms or other acute or chronic conditions of the lower respiratory tract.

b. Acute or chronic infection of the upper respiratory tract.

c. Anatomical nasal alterations, nasal polyposis, and other forms of non-allergic rhinitis.

d. History of use of a specific subcutaneous or sublingual immunotherapy with allergens.

e. Administration of topical or systemic steroids, antihistamines, leukotriene antagonists, and alpha-adrenergic antagonists (topical or systemic) in the past 4 weeks.

f. Active smokers or persons exposed to cigarette smoke in his/her family environment.

The minimum sample size was calculated to have a power of $80 \%$, a $95 \%$ confidence interval, for an estimated prevalence of "pure" allergic rhinitis of $30 \%$ over all forms of rhinitis, in 81 subjects applying the following formula: $n=z \alpha^{2}$ $\mathrm{p}(1-\mathrm{p}) / \mathrm{i}^{2}$.

Sampling was non-randomized and consecutive.

The study outcome measures included: age, sex, body mass index (BMI), and rhinitis duration. Tests included allergen skin tests with immediate reading using prick tests, a flow-volume curve spirometry, a nasal smear with eosinophil count, determination of total immunoglobulin E (IgE) by radioimmunoassay (RIA), and complete blood count with absolute and relative blood eosinophil count.
Rhinitis duration was valued in years based on the difference between age at the onset of symptoms and age at the moment of diagnosis. In order to make this outcome measure uniform, independently from age, the percentage of affected life was calculated using the following equation: [age at diagnosis - age at onset/age at diagnosis] x 100 .

For allergen skin tests, a standard panel with the following Alergo-Pharma ${ }^{\circledR}$ allergens was used: mites (Dermatophagoides pteronyssinus, D. farinae, Blomia tropicalis), environmental fungi (Alternaria, Aspergillus, Cladosporium, Mucor, Rhizopus, Penicillum species), dog epithelium, cat epithelium, mix of tree, grass and compound pollens, phenol-containing saline solution (negative control), and histamine $1 \mathrm{mg} / \mathrm{ml}$ (positive control). Tests were performed in the anterior side of the forearm with a pricker-like lancet (Diater Laboratorios ${ }^{\circledR}$ ), and the reading was done at 15 minutes using a millimeter graduated ruler. Results were considered positive if orthogonal diameter reading showed the presence of a papule of $>3 \mathrm{~mm}$ in average. ${ }^{16}$

The flow-volume curve was performed using

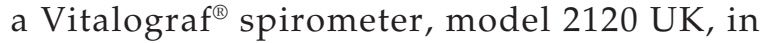
accordance with the international American Thoracic Society/European Respiratory Society (ATS/ERS) standards. ${ }^{17}$ The following parameters were determined: vital forced capacity (FVC), forced expiratory volume in 1 second of FVC (FEV1), Tiffeneau index (FEV1/FVC), and FEF $25-75 \%$, with values directly calculated using the software included in the device, as per Knudson's table of normal values. ${ }^{18}$ Values were considered abnormal if lower than $80 \%$ for the first three parameters, and lower than $70 \%$ for the FEF $25-$ $75 \%$ in relation to normal predictive values. The best three baseline determinations and the best three post-bronchodilator determinations were selected if they complied with the acceptability and reproducibility criteria as per the ATS/ERS standards. ${ }^{17}$

Nasal eosinophil count was determined analyzing nasal secretions sampled from the mucosal surface of the inferior turbinate with a plastic stylet with concave tip at the Anatomic Pathology Lab of the Clínica Universitaria Reina Fabiola. The May Grünwald-Giemsa staining method was used, and a value of $>5 \%$ was considered normal. ${ }^{19}$

The double-antibody radioimmunoassay (RIA) (Pharmacia, Uppsala ${ }^{\circledR}$, Sweden) technique 
was used to determine total serum IgE. Results were expressed in international units per milliliter (IU $/ \mathrm{mL}$ ). The absolute eosinophil count per cubic milliliter $\left(\mathrm{mm}^{3}\right)$ was determined in peripheral venous blood based on the relative percentage of total leukocytes in the complete blood count, with the May Grünwald-Giemsa staining method. The possibility of intestinal parasites was first ruled out using serial stool samples with anal smear, and other potential hypereosinophilic syndromes were ruled out based on the patient's medical record.

In all cases, the allergen skin tests, the flowvolume spirometry, the collection of samples for nasal smear, and the blood tests were performed by different operators who were unaware of the other study outcome measures. In order to reduce circadian variations, all complementary tests were done in the morning, between 9:00 AM and 12:00 PM.

\section{Ethical aspects}

The project was sent for evaluation to the Institutional Ethics Committee in Health Research (CIEIS) of Clínica Universitaria Reina Fabiola, and approved on December 27, 2011 by resolution $04 / 2011$. The research was done in compliance with the Declaration of Helsinki and the Good Clinical Practice standards, and in accordance with the Human Research Law of the Province of Cordoba No. 9694/09. The informed consent was obtained for all interventions performed and for the use of data, and confidentiality was warranted as per the Personal Data Protection Law No. 25326.

\section{STATISTICAL ANALYSIS}

Discrete outcome measures were described as percentage with $95 \%$ confidence intervals, and continuous outcome measures, as average, standard deviation, median and range. The associations between the different study factors and at least one altered spirometry function were analyzed using a multiple logistic regression model. The complete model was applied following the a posteriori elimination procedure of one non significant outcome measure at a time. A value of $\mathrm{p}<0.05$ was considered statistically significant.

\section{RESULTS}

During the study period, 4200 patients received care. Out of them, 102 were potentially eligible; 18 refused to participate and 84 male and female children and adolescents aged between 6 and 18 years old were included (51 males; 60\%), whose demographic and general characteristics are shown in Table 1. Pulmonary function values determined by the flow-volume curve are detailed in Table 2.

At least one altered respiratory function parameter was found in 21 out of the 84 studied patients (25\%; 95\% CI: 15.1-34.8). The FEV1/ FVC ratio was the most commonly affected parameter, either alone or combined with other functional outcome measure, while the FEF $25-75 \%$ was the least altered parameter (see Table 2).

Ten patients had only one compromised functional outcome measure: in five cases, the FEV1/FVC ratio; in four cases, the FVC; and in one case, the FEV1.

TABLE 1. Demographic characteristics and clinical and laboratory outcome measures in the study children and adolescents with allergic rhinitis

\begin{tabular}{lcccc}
\hline Outcome measure & n & Mean \pm SD & Median & Range \\
\hline Age (years) & 84 & $11.92 \pm 3.49$ & 11 & $6-18$ \\
Weight (kg) & 84 & $45.43 \pm 14.9$ & 44.50 & $19-81$ \\
Height (meters) & 84 & $1.52 \pm 0.16$ & 1.53 & $1.14-1.88$ \\
BMI & 84 & $19.07 \pm 3.01$ & 18.77 & $13.23-27.24$ \\
Rhinitis duration (in years) & 84 & $5.55 \pm 3.77$ & 4.65 & $0.25-17$ \\
Percentage of affected life & 84 & $45.88 \pm 25.18$ & 49 & $3-95$ \\
IgE (IU /ml) & 82 & $360.57 \pm 314.56$ & 241.50 & $16-1345$ \\
Blood eosinophils (x mm ${ }^{3}$ ) & 81 & $343.32 \pm 265.4$ & 292 & $0-1248$ \\
Blood eosinophils (\%) & 82 & $4.85 \pm 3.79$ & 4 & $0-24$ \\
Nasal smear eosinophils (\%) & 84 & $8.68 \pm 9.62$ & 5.60 & $0-48.4$ \\
\hline
\end{tabular}

SD: standard deviation.

$\mathrm{IU} / \mathrm{ml}$ : international units per milliliter. 
Of them, none developed an alteration in the FEF $25-75 \%$.

Eleven patients had alterations in more than one functional parameter. Out of the nine children and adolescents with two compromised parameters, 4 had a simultaneous alteration in the FEV1/FVC ratio and the FEF 25-75\%, four in the FVC and FEV1, and one in the FEV1 and FEF 25$75 \%$. Only two had three compromised functional outcome measures: one in relation to the FVC, FEV1/FVC and FEF $25-75 \%$, and one in relation to the FVC, FEV1 and FEF 25-75\%.

The multiple logistic regression analysis did not show any significant association between the pulmonary function alteration and the percentage of children with life affected by rhinitis (OR: 0.998; 95\% CI: $0.976-1.020 ; \mathrm{p}=0.86$ ), total serum lgE (OR: 0.999; 95\% CI: 0.996-1.001; $\mathrm{p}=0.41$ ), and nasal secretion eosinophils (OR: 0.982; 95\% CI: 0.640-1.508; $\mathrm{p}=0.93$ ).

Using the a posteriori elimination technique, the body mass index (OR: 1.31282; 95\% CI: $1.08611-1.58985 ; p=0.0049)$ and the absolute eosinophil count in peripheral blood (per $\mathrm{mm}^{3}$ ) (OR: $1.00229 ; 95 \%$ CI: $1.00022-1.00436 ; p=0.03$ ) were determined as significant predictive outcome measures for the presence of certain abnormal spirometry function.

\section{DISCUSSION}

The study showed that some patients with allergic rhinitis without asthma have alterations in the flow-volume curve. Although some anecdotal reports have described the relation between rhinitis and asthma, the physiological and pathological connections between the upper and lower airways have only been recognized in the past twenty years, now known as a "single airway."2-4 Since then, the nose and the lungs are considered closely related organs from an epidemiological, anatomical and functional perspective, with resulting pathological implications. ${ }^{2}$

Epidemiological studies have witnessed a high rate of these two conditions taking place concomitantly, and rhinitis is often the predecessor of allergic asthma. ${ }^{1,20}$ In patients with allergic rhinitis, signs of allergic inflammation in the lower airway have been confirmed by induced sputum, bronchoalveolar lavage, and endobronchial biopsy., ${ }^{3,21}$ As a result, subclinical alterations in the pulmonary function and increased reactivity to bronchodilator response could occur in adults ${ }^{7}$ and children ${ }^{8-10}$ with allergic rhinitis, which could have diagnostic, prognostic and therapeutic consequences.

Our research detected the presence of subclinical functional alterations in children with allergic rhinitis with no symptoms of clinically evident asthma. A high percentage of study children and adolescents had an alteration in at least one of the flow-volume curve spirometry parameters. Unlike other studies, ${ }^{7-10}$ we observed that the FEV1/FVC ratio was the most commonly affected parameter, either alone or combined with other functional outcome measure. A reduction in the Tiffeneau index is the preferred parameter for diagnosing intrathoracic airway obstruction; $; 1,17$ therefore, it is recognized that the children with rhinitis in our study who showed an altered FEV1/FVC ratio have an obstructive functional involvement.

TABLE 2. Pulmonary function, frequency and characteristics of alterations in functional respiratory parameters for non standard cases

\begin{tabular}{|c|c|c|c|c|c|c|}
\hline $\begin{array}{l}\text { Outcome } \\
\text { measure }\end{array}$ & $\mathbf{n}$ & $\begin{array}{r}\text { Total number of } \\
\text { cases Mean } \pm \text { SD }\end{array}$ & Range & $\mathbf{n}$ & $\begin{array}{l}\text { In standard cases } \\
\text { Mean } \pm \text { SD }\end{array}$ & Range \\
\hline$\overline{\mathrm{FVC}(\mathrm{L})}$ & 84 & $2.94 \pm 1.09$ & $1.30-6.20$ & 9 & $2.56 \pm 0.59$ & $1.81-3.46$ \\
\hline FVC $(\%)^{*}$ & 84 & $95.71 \pm 13.32$ & $71.00-133.70$ & 9 & $75.44 \pm 2.64$ & 71-78.3 \\
\hline FEV1 (L) & 84 & $2.58 \pm 0.90$ & $1.20-4.95$ & 8 & $2.15 \pm 0.57$ & $1.57-3.31$ \\
\hline FEV1 (\%)* & 84 & $95.41 \pm 11.93$ & $64.40-125$ & 8 & $75.75 \pm 5.01$ & $64.4-79.8$ \\
\hline FEV1/FVC* & 84 & $88.60 \pm 6.23$ & $67.80-108.20$ & 10 & $76.63 \pm 3.38$ & $67.8-79.8$ \\
\hline FEF 25-75 (L/s) & 84 & $2.95 \pm 0.99$ & $1.06-4.98$ & 7 & $2.13 \pm 0.74$ & $1.06-3.16$ \\
\hline FEF $25-75(\%)^{* *}$ & 84 & $95.27 \pm 19.46$ & $37.60-141.10$ & 7 & $59.67 \pm 10.23$ & $37.6-69$ \\
\hline
\end{tabular}

L: liter; L/s: liter per second. SD: standard deviation.

* Values were considered abnormal if lower than $80 \%$ in relation to predictive values as per Knudson's table of normal values. ${ }^{18}$

** Values were considered abnormal if lower than $70 \%$ in relation to predictive values as per Knudson's table of normal values. ${ }^{18}$ 
Although the flow-volume curve does not provide direct spirometry parameters capable of evaluating the small airway, the FEF $25-75 \%$ is considered to have a higher sensitivity than the FEV1 for the determination of the initial stages of involvement in the most peripheral airway of low caliber. ${ }^{14,15}$ In our study, the FEF $25-75 \%$ was the least affected parameter, probably because the alteration threshold in our study was a value equal to or lower than $70 \%$, unlike other authors, who used a threshold of less than $80 \% .{ }^{7,8}$

No alterations were found in any of the four functional outcome measures in any case, but two children had three altered parameters, thus reinforcing the possibility of a more extended airway involvement in these patients. It is striking that four patients only showed a FVC alteration, indicating a restrictive involvement of their pulmonary function; this is probably due to the impact on the breathing capacity of the chest musculature secondary to the nasal obstruction typical of patients with persistent rhinitis and not to an intrinsic pulmonary defect caused by the systemic allergic inflammation. Our results allow us to suggest that the recommendations made in the ARIA (Allergic Rhinitis and its Impact on Asthma $)^{2}$ document for indicating a spirometry assessment for each patient with allergic rhinitis should include children and adolescents with the same clinical condition. In addition, it would have possible therapeutic implications. Patients with allergic rhinitis and subclinical bronchial obstruction could benefit from a treatment for their condition. Such assumption is sustained by a recent study indicating that in an important number of perennial allergic rhinitis patients with reduced FEF $25-75 \%$ values, such functional disorder was significantly reverted with the administration of endonasal budesonide for three months. ${ }^{9}$ In our study, research included the factors that may influence the presence of these alterations on pulmonary function and we observed that two outcome measures are associated with an altered respiratory function: blood eosinophils (absolute value per $\mathrm{mm}^{3}$ ) and body mass index. Given their OR, the relationships are positive: with a higher BMI and peripheral blood eosinophil count, the higher is the probability of having at least one altered pulmonary function outcome measure.

Although it has been previously demonstrated that higher values of total serum IgE and nasal secretion eosinophils have been associated with bronchial hiperreactivity to methacholine in children with allergic rhinitis without asthma, the present study has not shown that these outcome measures can be significant predictors of the alterations observed in the flow-volume curve. Previous studies detected an increase in the eosinophil lineage and in the expression of related cytokines (IL-5) in bone marrow and peripheral blood following a nasal challenge test with allergens, ${ }^{21,22}$ indicating that blood eosinophilia is probably more related to systemic inflammation than nasal eosinophilia and serum $\operatorname{IgE}$, with an impact on the intrathoracic airway and the resulting subclinical involvement of the pulmonary function. The influence of BMI on pulmonary functional outcome measures in our patients is somewhat more difficult to explain. A recent study ${ }^{23}$ provides evidence of a significant correlation between BMI and allergic rhinitis and bronchial hyperreactivity, but no evidence related to the impact on the functional parameters assessed in the spirometry. Although evidence is not unanimous, ${ }^{24,25}$ body mass index indicators suggestive of obesity can affect the respiratory function, with a reduction in the pulmonary volume and capacity in the absence of a respiratory condition, and can exaggerate such effect on a pre-existing airway condition. At the same time, a higher BMI could cause mechanical consequences due to the involvement of the adaptability of the chest wall, and probably, immune changes induced by adipokines and cytokines secreted by adipose tissue, which can produce proinflammatory changes. ${ }^{26}$ Such speculations should be confirmed by studies conducted in children, especially because it is known that the inflammatory phenotype associated with obesity is a neutrophilic (not eosinophilic) phenotype, as would be assumed with our allergic patients.

It has been suggested that the duration of nasal allergic conditions is directly related to the severity of pulmonary function involvement. 7,8 Our study results contrast such evidence. In this study, we assessed the impact of the percentage of life affected by the disease and found no influence on pulmonary function, consistent with a recent study evaluating rhinosinusitis and nasal polyposis on the pulmonary function of adults. ${ }^{13}$

One of the strengths of this study is that it included children and adolescents with allergic rhinitis without asthma, a population poorly studied in the medical literature; in addition, potentially confounding outcome measures were studied, such as weight, age, symptom duration, 
and BMI using a multiple logistic regression model and adjusting such outcomes.

We recognize that the analysis method used here could have limitations for establishing risk factors; therefore, future studies will allow to deepen the knowledge of airway physiology as a unit and to detect the definitive functional impact of allergic rhinitis on the lower airway and its potential therapeutic implications.

\section{CONCLUSIONS}

Our study data demonstrate the presence of respiratory functional alterations in many children with allergic rhinitis without asthma; the FEV1/FVC ratio is the most commonly affected parameter. The absolute blood eosinophil count and the body mass index may be associated with a sub-clinical alteration in the pulmonary function of these patients.

\section{REFERENCES}

1. Ait-Khaled N, Pearce N, Anderson HR, Ellwood P, Montefort S, Shah J, and the ISAAC Phase Three Study Group. Global map of the prevalence of symptoms of rhinoconjunctivitis in children: The International Study of Asthma and Allergies in Childhood (ISAAC) Phase Three. Allergy 2009;64:123-48.

2. Bousquet J, Khaltaev N,CruzAA, etal.Allergic Rhinitis and its impact on asthma (ARIA). 2008 Update (in collaboration with the World Health Organization, GA2LEN and AllerGen). Allergy 2008;63:S8-S160.

3. Braunstahl GJ. United airways concept: what does it teach us about systemic inflammation in airways disease? Proc Am Thorac Soc 2009;6:652-4.

4. Saranz RJ. Vías aéreas unidas e impacto recíproco entre rinosinusitis y asma. Alergia Immunol Clin 2002;19(3):71-77.

5. Saranz RJ, Lozano A, Alvarez J, Croce JS, et al. Factors associated to bronchial hyperresponsiveness in children and adolescents with allergic rhinitis. J Allergy Clin Immunol 1997;99(1):S418.

6. Lozano A. Aportes a la Fisiopatogenia de la Rinitis Alérgica: Rinitis inducida por ejercicio. Tesis Doctoral. Facultad de Medicina. Universidad Católica de Córdoba. Marzo 2002.

7. Ciprandi G, Cirillo I, Pistorio A. Impact of allergic rhinitis on asthma: effects on spirometric parameters. Allergy 2008;63:255-60.

8. CiprandiG,Capasso M.Association of childhood perennial allergic rhinitis with subclinical airflow limitation. Clin Exp Allergy 2010;40:398-402.

9. Kessel A, Halloun H, Bamberger E, Kugelman A, Toubi E. Abnormal spirometry in children with persistent allergic rhinitis due to mite sensitization: The benefit of nasal corticosteroids. Pediatr Allergy Immunol 2008;19:61-66.

10. Capasso M, Varricchio A, Ciprandi G. Impact of allergic rhinitis on asthma in children: effects on bronchodilation test. Allergy 2010;65:264-8.

11. Ramsdale EH, Morris MM, Roberts RS, Hargreave FE. Asymptomatic bronchial hyperresponsiveness in rhinitis. J Allergy Cin Immunol 1985;75:573-577.

12. Muller BA, LeickCA, Smith RM, Suelzer MT, Richerson HB. Comparison of specific and nonspecificbronchoprovocation in subjects with asthma, rhinitis and healthy subjects. J Allergy Clin Immunol 1993;91:758-72.

13. Williamson PA, Vaidyanathan S, Clearie K, Barnes M. Lipworth B.J. Airway dysfunction in nasal polyposis: a spectrum of asthmatic disease? Clin Exp Allergy 2011;41:1379-85.

14. Wallace DV, Dykewicz MS. The diagnosis and management of rhinitis: an updated practice parameter. J Allergy Clin Immunol 2008;122:S1-S84.

15. Pellegrino R, Viegi G, Brusasco V, Crapo RO, et al. Interpretative strategies for lung function tests. Eur Respir J 2005;26:948-68.

16. Cox L, Williams B, Sicherer S, Oppenheimer J, Sher L, et al. Pearls and pitfalls of allergy diagnostic testing: report from the American College of Allergy, Asthma and Immunology / American Academy of Allergy, Asthma and Immunology Specific IgE Test Task Force. Ann Allergy Asthma Immunol 2008;101:580-92.

17. Miller MR, Hankinson J, Brusasco V, Burgos F,et al. Standardisation of spirometry. Eur Respir J 2005;26:319-38.

18. Knudson RJ, Slatin RC, Lebowitz MD, Burrows B. The maximal expiratory flow-volume curve. Normal standards, variability, and effects of age. Am Rev Respir Dis 1976;113:587-600.

19. Mygind N. Leucocitos y eosinófilos. De Mygind N. Alergia Nasal; 2da ed., Salvat Eds. Barcelona, España; 1982. Págs.171-182.

20. LeynaertB,NeukirchF,DemolyP,BousquetJ.Epidemiologic evidence for asthma and rhinitis comorbidity. J Allergy Clin Immunol 2000;106:S201-5.

21. Togias AG. Systemic immunologic and inflammatory aspects of allergic rhinitis. J Allergy Clin Immunol 2000;106(5 Suppl):S247-50.

22. Dorman SC, Sehmi R, Gauvreau GM, et al. Kinetics of bone marrow eosinophilopoiesis and associated cytokines after allergen inhalation. Am J Respir Crit Care Med 2004;169(5):565-72.

23. Ciprandi G, Pistorio A, Tosca M, Ferraro MR, Cirillo I. Body mass index, respiratory function and bronchial hyperreactivity in allergic rhinitis and asthma. Respiratory Med 2009;103:289-95.

24. Salome CH, King GG, Berend N. Physiology of obesity and effects on lung function. J Appl Physiol 2010;108:206-11.

25. Littleton SW. Impact of obesity on respiratory function. Respirology 2012;17:43-9.

26. Fantuzzi G. Adipose tissue, adipokines, and inflammation. J Allergy Clin Immunol 2005;115:911-919. 